\title{
Multigene assays and molecular markers in breast cancer: systematic review of health economic analyses
}

\author{
Roman Rouzier • Paolo Pronzato · Elisabeth Chéreau • \\ Josh Carlson • Barnaby Hunt $\cdot$ William J. Valentine
}

Received: 16 April 2013/Accepted: 30 April 2013/Published online: 31 May 2013

(C) The Author(s) 2013. This article is published with open access at Springerlink.com

\begin{abstract}
Breast cancer is the most common female cancer and is associated with a significant clinical and economic burden. Multigene assays and molecular markers represent an opportunity to direct chemotherapy only to patients likely to have significant benefit. This systematic review examines published health economic analyses to assess the support for adjuvant therapy decision making. Literature searches of PubMed, the Cochrane Library, and congress databases were carried out to identify economic evaluations of multigene assays and molecular markers published between 2002 and 2012. After screening and data extraction, study quality was assessed using the Quality of Health Economic Studies instrument. The review identified 29 publications that reported evaluations of two assays: Oncotype DX ${ }^{\circledR}$ and MammaPrint. Studies of both tests provided evidence that their routine use was cost
\end{abstract}

\author{
R. Rouzier \\ Institut Curie, 26 rue d'Ulm, 75005 Paris, Saint Cloud, France \\ e-mail: roman.rouzier@curie.fr \\ R. Rouzier \\ EA 7285, UVSQ, Versailles, France \\ R. Rouzier · E. Chéreau \\ Institut Paoli Calmette, Marseille, France \\ P. Pronzato \\ IRCCS Azienda Ospedaliera, Universitaria San Martino, Genoa, \\ Italy \\ J. Carlson \\ University of Washington, Seattle, WA, USA \\ B. Hunt · W. J. Valentine $(\bowtie)$ \\ Ossian Health Economics and Communications, Basel, \\ Switzerland \\ e-mail: valentine@ossianconsulting.com
}

saving or cost-effective versus conventional approaches. Benefits were driven by optimal allocation of adjuvant chemotherapy and reduction in chemotherapy utilization. Findings were sensitive to variation in the frequency of chemotherapy prescription, chemotherapy costs, and patients' risk profiles. Evidence suggests that multigene assays are likely cost saving or cost-effective relative to current approaches to adjuvant therapy. They should benefit decision making in early-stage breast cancer in a variety of settings worldwide.

Keywords Breast cancer - Gene expression profiling . Gene assay - Molecular diagnostic techniques .

Health economics · Cost-effectiveness

\author{
Abbreviations \\ ER Estrogen receptor \\ HTA Health Technology Assessment \\ LN Lymph node \\ MeSH Medical subject heading \\ MGA Multigene assay \\ NCCN National Comprehensive Cancer Network \\ NICE National Institute for Health and Clinical Excellence \\ NIH National Institutes of Health \\ QALE Quality-adjusted life expectancy \\ QALY Quality-adjusted life year \\ QHES Quality of Health Economic Studies \\ QoL Quality of life \\ SABC San Antonio Breast Cancer
}

\section{Introduction}

Breast cancer places a substantial burden on healthcare providers and is associated with significant mortality and reduced 
quality of life (QoL) [1-5]. Currently, following curativeintent, patients typically undergo adjuvant treatment consisting of radiotherapy, systematic treatment (if the tumor is sensitive), human-epidermal-growth-factor-receptor2 (HER2)-directed therapy (if HER2 is overexpressed), and often chemotherapy. The aim is to avoid cancer recurrence and improve overall survival. Clinicians face difficult decisions when prescribing adjuvant therapy, particularly with regard to chemotherapy, balancing the benefit in terms of reduced risk of recurrence, and improved survival with the adverse effects of treatment. Current guidelines recommend chemotherapy in the majority of early-stage breast cancer patients.

Recent development of commercially available multigene assays (MGAs) may represent an opportunity to identify patients who will and will not benefit from chemotherapy, and adapt prescriptions accordingly. MGAs quantify the expression of genes associated with the underlying tumor biology and long-term outcomes [6-8]. Assays have proved to be prognostic and there are data supporting prediction of chemotherapy benefit $[9,10]$. MGAs have been included in the major international guidelines for adjuvant breast cancer treatment in recent years [11-14]. This systematic review summarizes the available evidence from health economic analyses on MGAs and molecular markers in breast cancer.

\section{Literature search}

Articles evaluating the economic impact of commercially available MGAs and protein expression profiling on prescriptions of adjuvant systemic therapy in breast cancer were identified via a literature search. The tests included were technologies under consideration by NICE in a recent scoping exercise (Oncotype DX ${ }^{\circledR}$, MammaPrint, Blueprint, PAM50, Breast Cancer Index, Mammostrat, and NPI+) $[15,16]$. The target population was considered to be all early-stage, nonmetastatic breast cancer patients who underwent curative-intent surgery.

Literature search of PubMed and the Cochrane Library used medical subject heading (MeSH) major topics and a number of "title and abstract" searches combined with Boolean operators, to identify economic evaluations of molecular diagnostic tests published between 1/1/2002 and 1/7/2012 [17]. Hand searches of PubMed identified recently published articles without assigned MeSH terms. HTA authority websites of the UK, Canada, Australia, and USA were searched for any reports containing economic models pertinent to this review. Meeting presentations between 2009 and 2012 were also searched (Appendix).

Citations were screened by title and abstract against inclusion criteria to identify publications assessing the cost-effectiveness or budget impact of prognostic MGAs and molecular markers. Full text was obtained for publications not clearly classified by abstract review. From included articles, data were extracted on the methodological characteristics and results of the publications. Methodological data included the country setting, year of analysis, modeling approach, time horizon, population, outcomes reported, and data sources used. Extracted results data were clinical outcomes, cost outcomes, and costeffectiveness results. In addition, studies evaluating budget impact were included, defined as the estimation of the financial consequences of introducing new healthcare interventions for a defined healthcare payer or system.

The validated [18, 19] Quality of Health Economic Studies (QHES) instrument was used to evaluate the quality of economic evaluations. The QHES instrument consists of 16 criteria addressing methodological characteristics and transparency of reporting, and against which economic evaluations are compared.

\section{Results}

\section{Study selection}

From 572 unique articles, screening identified 14 publications assessing the economic impact of using MGAs to guide adjuvant breast cancer therapy. Hand searching of PubMed identified four additional articles that had not yet been assigned MeSH terms and the congress database search yielded 14 pertinent abstracts. Of these, two studies were described in journal articles already identified and were therefore excluded. One further article was excluded following full text review because no price for the genomicprofiling test was included [20]. Consequently, the 29 studies reviewed here comprise 17 journal published manuscripts and 12 abstracts (Fig. 1). Of the identified studies, the majority compared a MGA with current practice, either Adjuvant!, St. Gallen or NCCN guidelines. Oncotype $\mathrm{DX}^{\circledR}$ was the test most commonly evaluated, with 18 costeffectiveness analyses and four budget impact studies comparing it with current practice [21-42]. Of the remaining studies, four cost-effectiveness evaluations and one budget impact study compared MammaPrint with current practice, and two studies compared Oncotype $\mathrm{DX}^{\circledR}$ with MammaPrint [43-49]. Table 1 provides a brief description of Oncotype DX $^{\circledR}$ and MammaPrint, the two tests with identified published economic literature for review.

\section{Cost-effectiveness evaluations of Oncotype $\mathrm{DX}^{\circledR}$} versus usual care

A total of 18 studies were identified that evaluated the costeffectiveness of Oncotype $\mathrm{DX}^{\circledR}$ versus current treatment in 


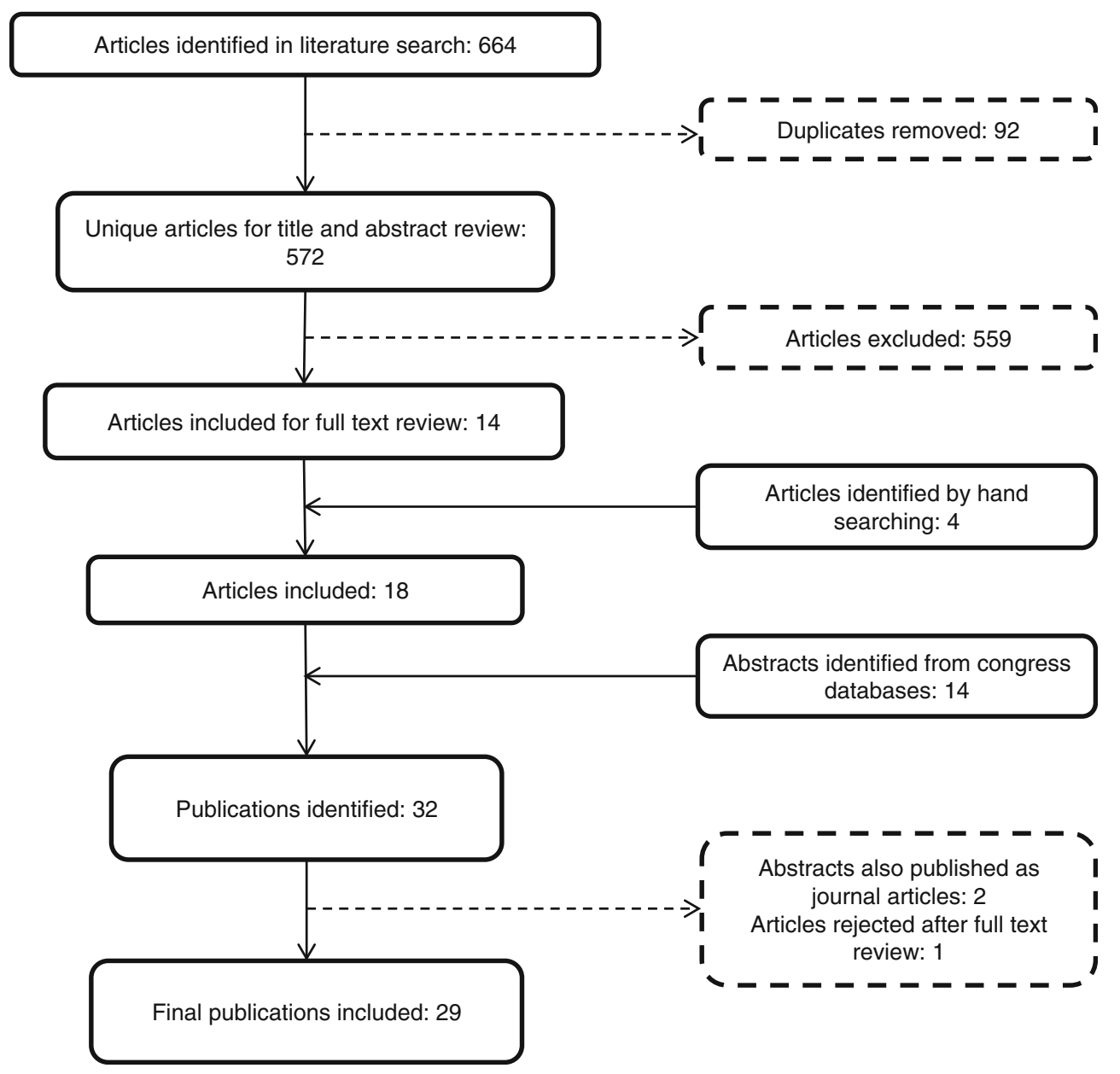

Fig. 1 CONSORT flow diagram of literature review

estrogen receptor positive $(\mathrm{ER}+)$, HER2- early breast cancer (Table 2). Of the 18 cost-effectiveness studies identified, four explicitly stated that they used the same model structure developed by Hornberger et al. in the first published cost-effectiveness evaluation on Oncotype $\mathrm{DX}^{\circledR}$ [21-23, 32, 33], while a further four used a similar modeling approach [24-26, 34]. The model developed by Hornberger et al. [21] utilized a decision tree approach to model which adjuvant therapies patients received before and after Oncotype $\mathrm{DX}^{\circledR}$ testing. Outcomes were then simulated based on data from landmark trials using a Markov model consisting of four states; no recurrence, recurrence with no metastatic progression, recurrence with metastatic progression, and death. The impact of chemotherapy (in terms of reduced risk of distant recurrence, decreased QoL, and increased costs) was estimated in the Markov model, depending on the treatment allocated in the decision tree. Conceptually, a similar approach was used in all of the Oncotype $\mathrm{DX}^{\circledR}$ cost-effectiveness modeling studies identified in this review (i.e., estimates of long-term outcomes in a population with and without Oncotype $\mathrm{DX}^{\circledR}$ testing).
The results of the published cost-effectiveness analyses were broadly consistent across the countries investigated (including Australia, Canada $(n=5)$, Hungary, Ireland $(n=3)$, Israel, Japan $(n=2)$, Singapore $(n=2)$, UK $(n=2)$, and USA $(n=6))$, indicating that Oncotype $\mathrm{DX}^{\circledR}$ testing is likely to improve outcomes, reduce the proportion of patients treated with chemotherapy, and be cost-effective from a healthcare payer perspective according to commonly accepted cost-effectiveness thresholds. Results were different in the USA where Oncotype $\mathrm{DX}^{\circledR}$ not only improved outcomes, but was also cost saving (i.e., dominant to the current standard of care), an effect driven largely by the fact that chemotherapy is more frequently recommended and more expensive in the USA. This was reflected in the recent analysis by Hornberger et al. [32] based on chemotherapy utilization data from a meta-analysis of decision impact studies, which suggested that Oncotype $\mathrm{DX}^{\circledR}$ guided decision making dominated chemotherapy decision making based on NCCN guidelines. Other studies based on real-life chemotherapy prescribing rates have shown Oncotype $\mathrm{DX}^{\circledR}$ to be cost-effective 
Table 1 Description of interventions included in the review

\begin{tabular}{lll}
\hline Intervention & Level of supporting clinical evidence & Description, prognostic and predictive ability \\
\hline
\end{tabular}

Oncotype $\mathrm{DX}^{\circledR} \quad 9$ studies on analytical validation

8 publications describing clinical validation, of which 7 studies provided evidence on the prognostic ability, 1 study provided data on predictive ability and 1 study provided results on both

11 publications on decision impact studies

MammaPrint

6 publications describing analytical/clinical validation, of which publication provided data on prognostic ability (predictive ability is assumed by association)

No decision impact studies were identified
The assay evaluates the expression of a panel of 21 genes from a tumor specimen (biopsy) using a high-throughput, real-time reverse-transcriptase polymerase chain reaction (RT-PCR) method to measure levels of gene expression. The gene expression results from the assay are combined into a single score called the Recurrence Score, which corresponds to a point estimate of the 10-year risk of distant recurrence with a $95 \%$ confidence interval for an individual patient (expressed as a value between 0 and 100).

Seven clinical trials have shown that Oncotype $\mathrm{DX}^{\circledR}$ provides a reliable evaluation of the risk of distant recurrence in early-stage breast cancer patients. Studies have shown that low Recurrence Score disease is associated with both a lower risk of distant recurrence (as well as little (or no) chemotherapy benefit). From a physician-patient perspective, the risk of distant recurrence (based on the Recurrence Score) may directly influence chemotherapy decision-making, with patients showing a high risk of recurrence choosing adjuvant chemotherapy. For patients with Recurrence Scores in the intermediate range (between 18 and 30 ), having a risk of recurrence in the upper end of the intermediate range may lead to an increased interest in choosing chemotherapy compared with having a risk of recurrence in the lower end of the intermediate range.

The ability of Oncotype $\mathrm{DX}^{\circledR}$ to predict the potential benefit of adjuvant chemotherapy has been directly validated using two prospectively designed studies of archived tumor specimens from well-controlled clinical studies. These studies provide clear evidence that patients with low Recurrence Scores derived minimal, if any, benefit from chemotherapy in addition to endocrine therapy and those with high Recurrence Scores had a substantial benefit from the addition of chemotherapy to adjuvant endocrine treatment. Further, data from two exploratory neoadjuvant trials where complete pathological responses (Gianni et al. 2005) or complete clinical response (Chang et al. 2008) have only been seen in patients with a Recurrence Score of 25 or higher has emphasized the potential utility of the Recurrence Score in predicting chemotherapy benefits

The assay utilizes microarray technology to evaluate the expression of a 70-gene panel, generated using RT-PCR, in fresh/frozen tissue from test and reference samples. After normalization of results, computer analysis is performed on the microarray results of normal and diseased tissue can be compared to identify genes that vary in their expression and also identify a pattern (profile) that may indicate a distinct class or stage of disease. Based on these results, patients are classified as at a high or low risk of 5 -year distant recurrence.

One clinical study has provided evidence that the MammaPrint gene signature has the ability to identify the likelihood of distant recurrence in the first 5 years following diagnosis. Based on the observation from the Early Breast Cancer Trialists' Collaborative Group, that adjuvant chemotherapy exerts its principal benefit in reducing early metastasis risk during the first 5 years, it is assumed that MammaPrint is predictive during the same interval over which adjuvant chemotherapy exerts the maximum benefit.

Evidence regarding the prediction of benefits from chemotherapy has not been independently demonstrated in randomized trials

Information on Oncotype $\mathrm{DX}^{\circledR}$ was derived from the Manufacturer/Sponsor Submission of Evidence on the Oncotype DX ${ }^{\circledR}$ Breast Cancer Test to NICE in 2011. Information on the MammaPrint test was taken from the product website (http://www.agendia.com/pages/mammaprint/21.php) accessed on August 16,2012 


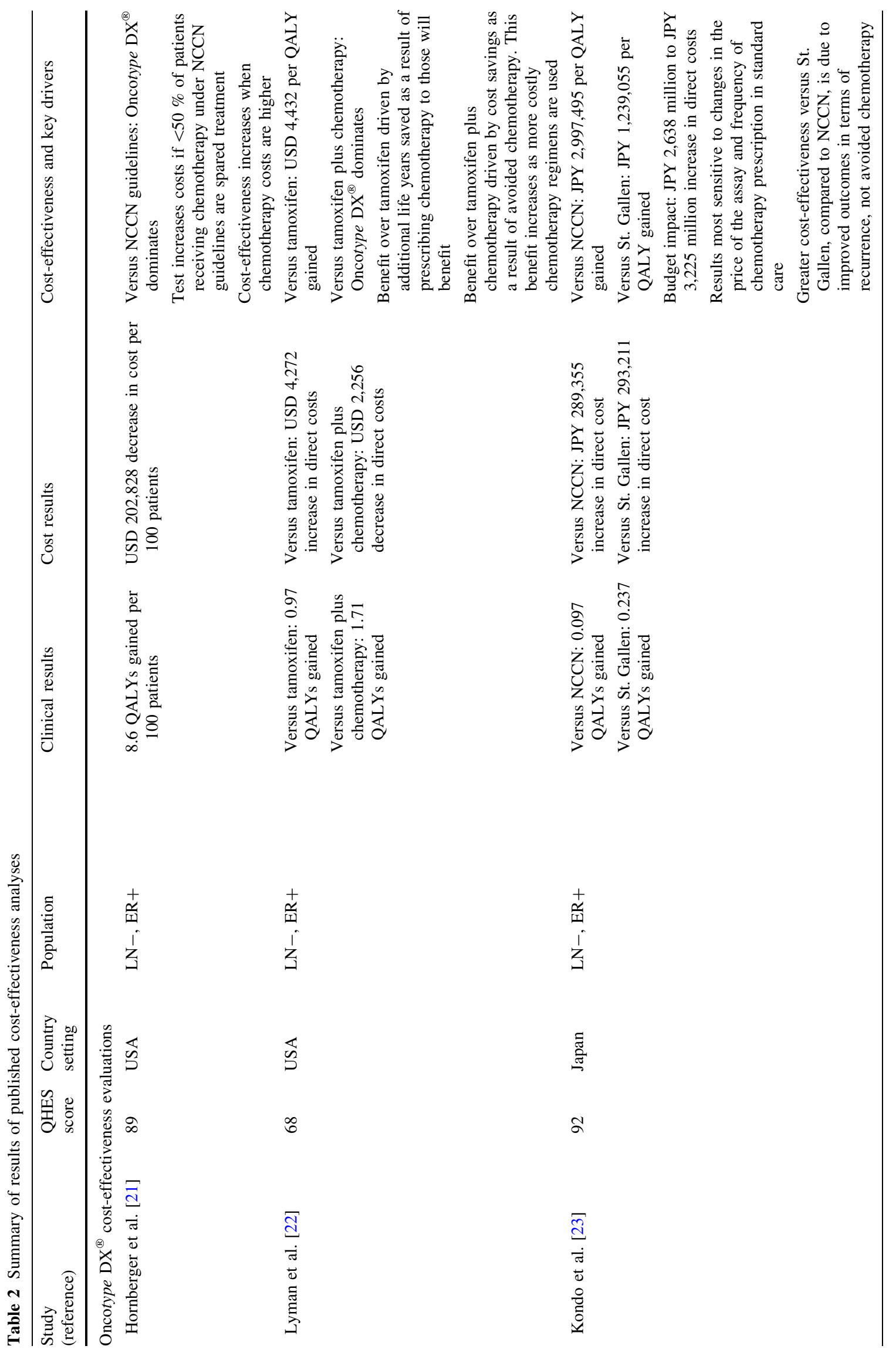




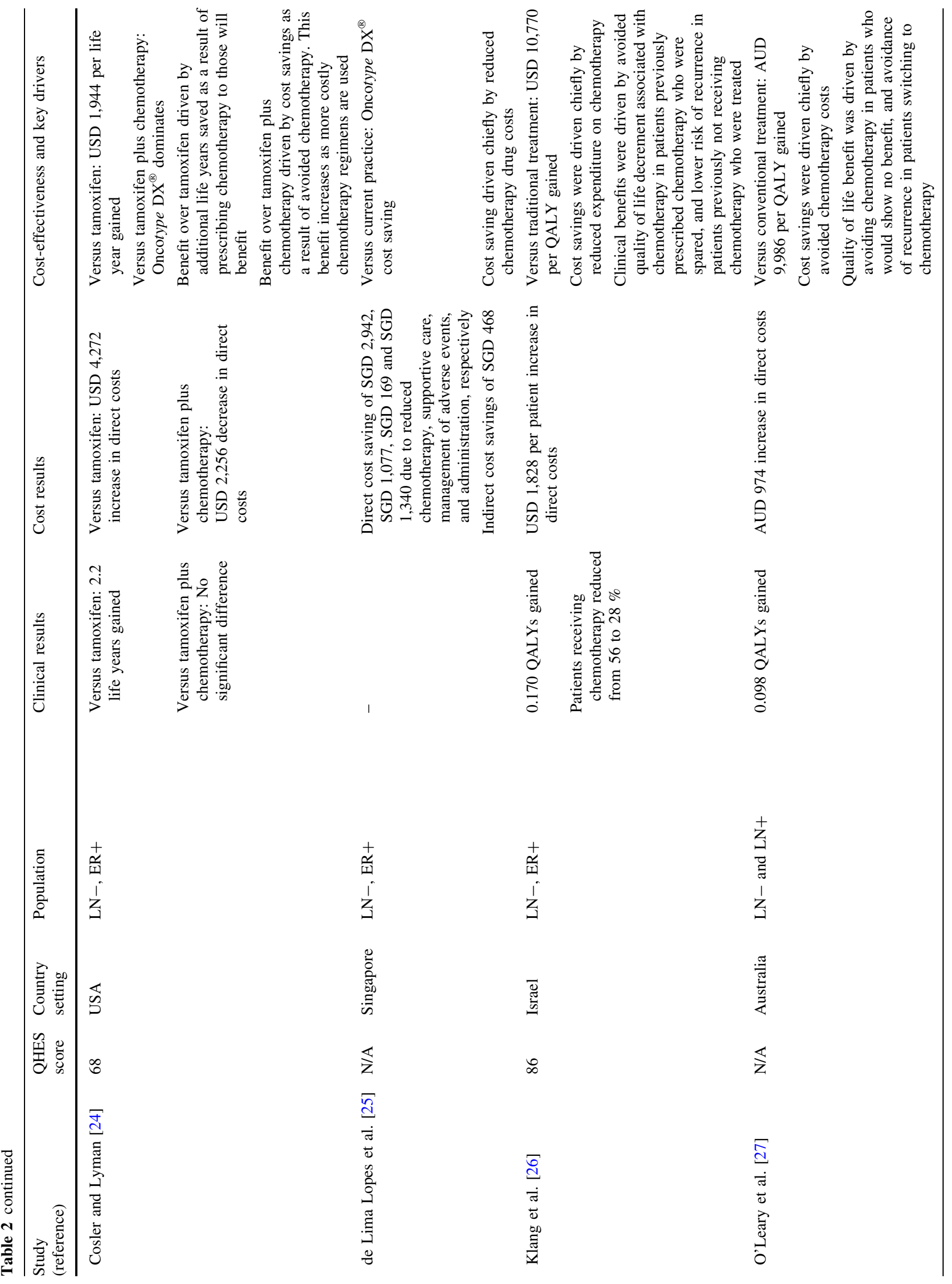




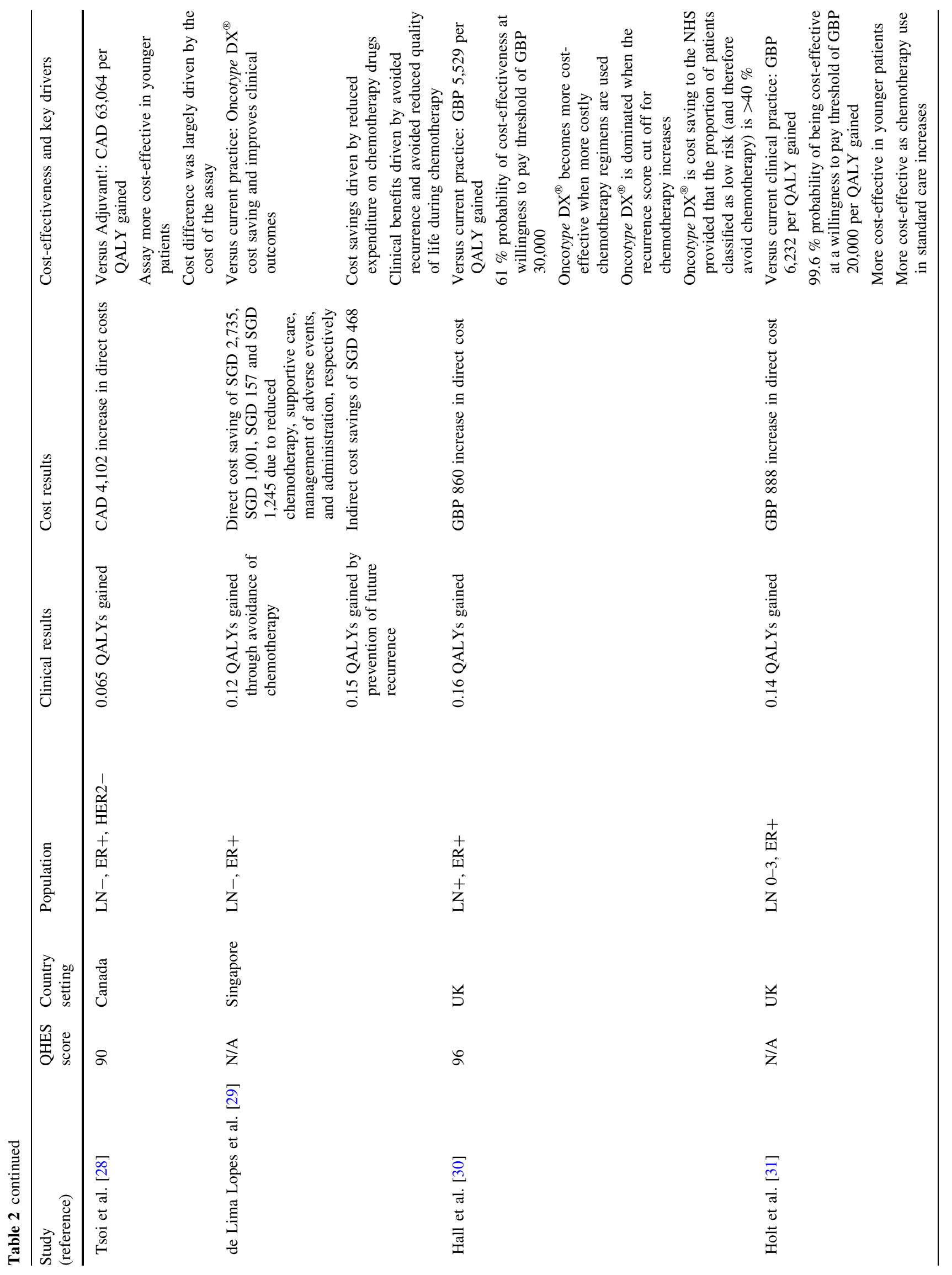




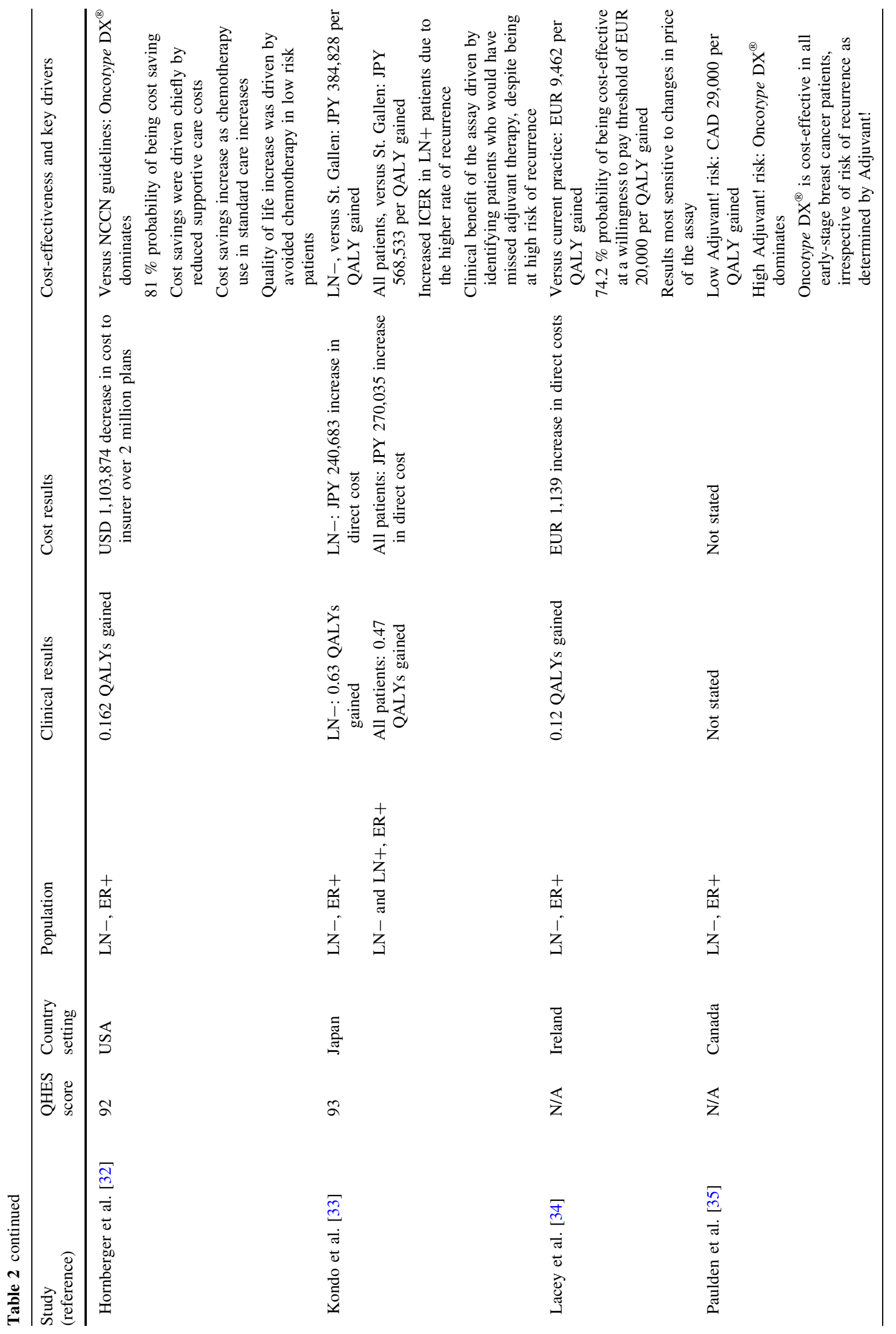




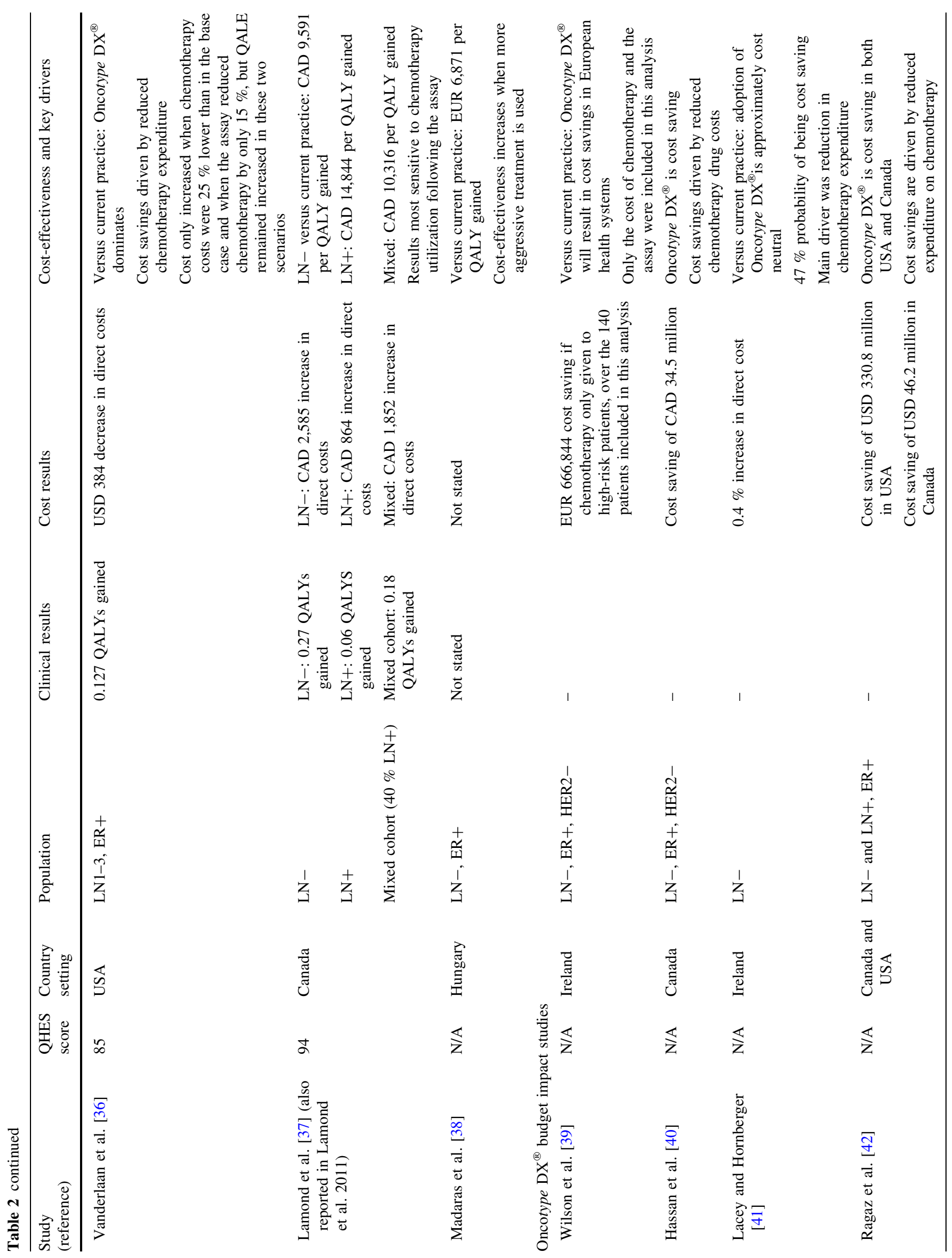




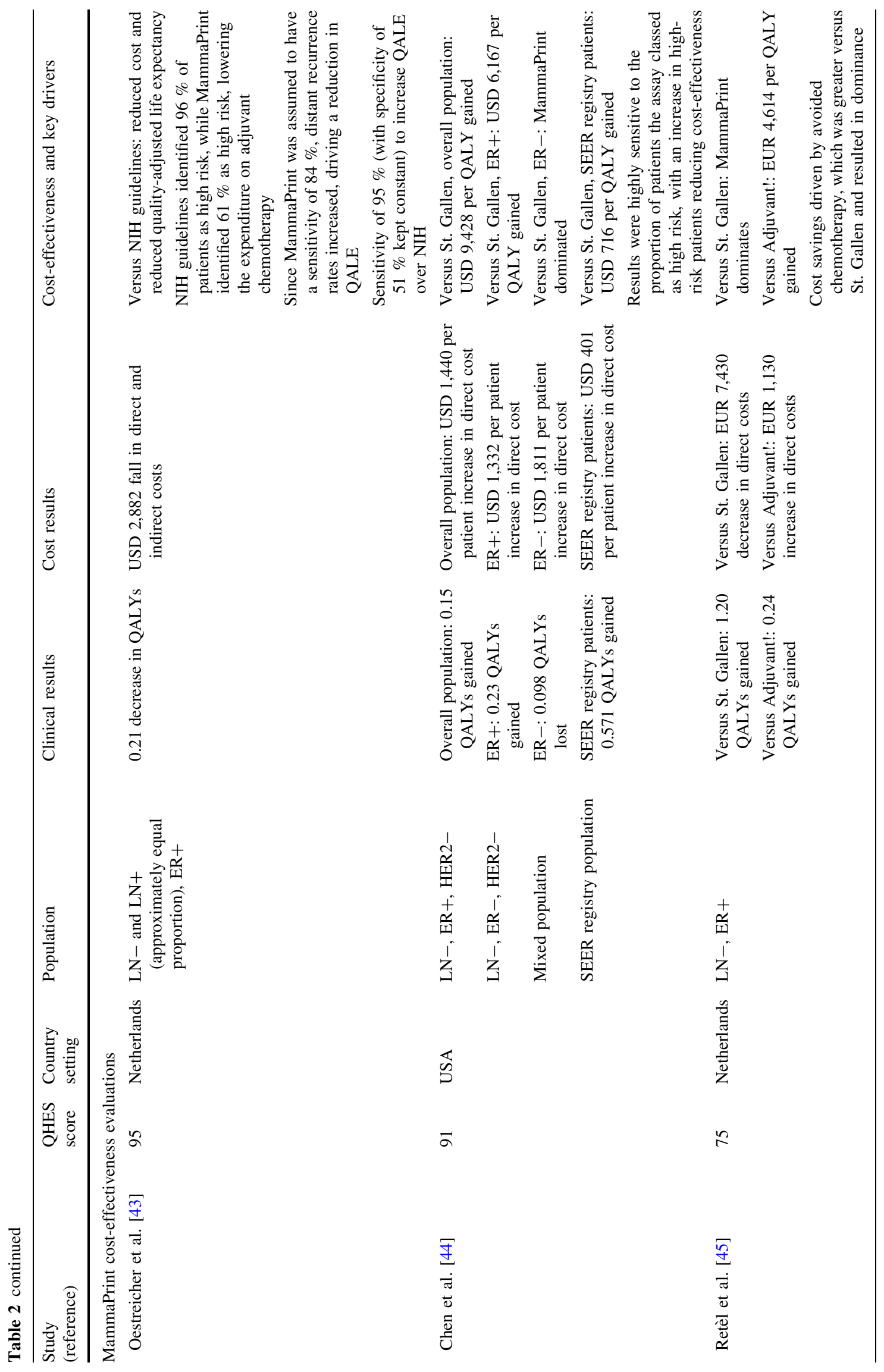




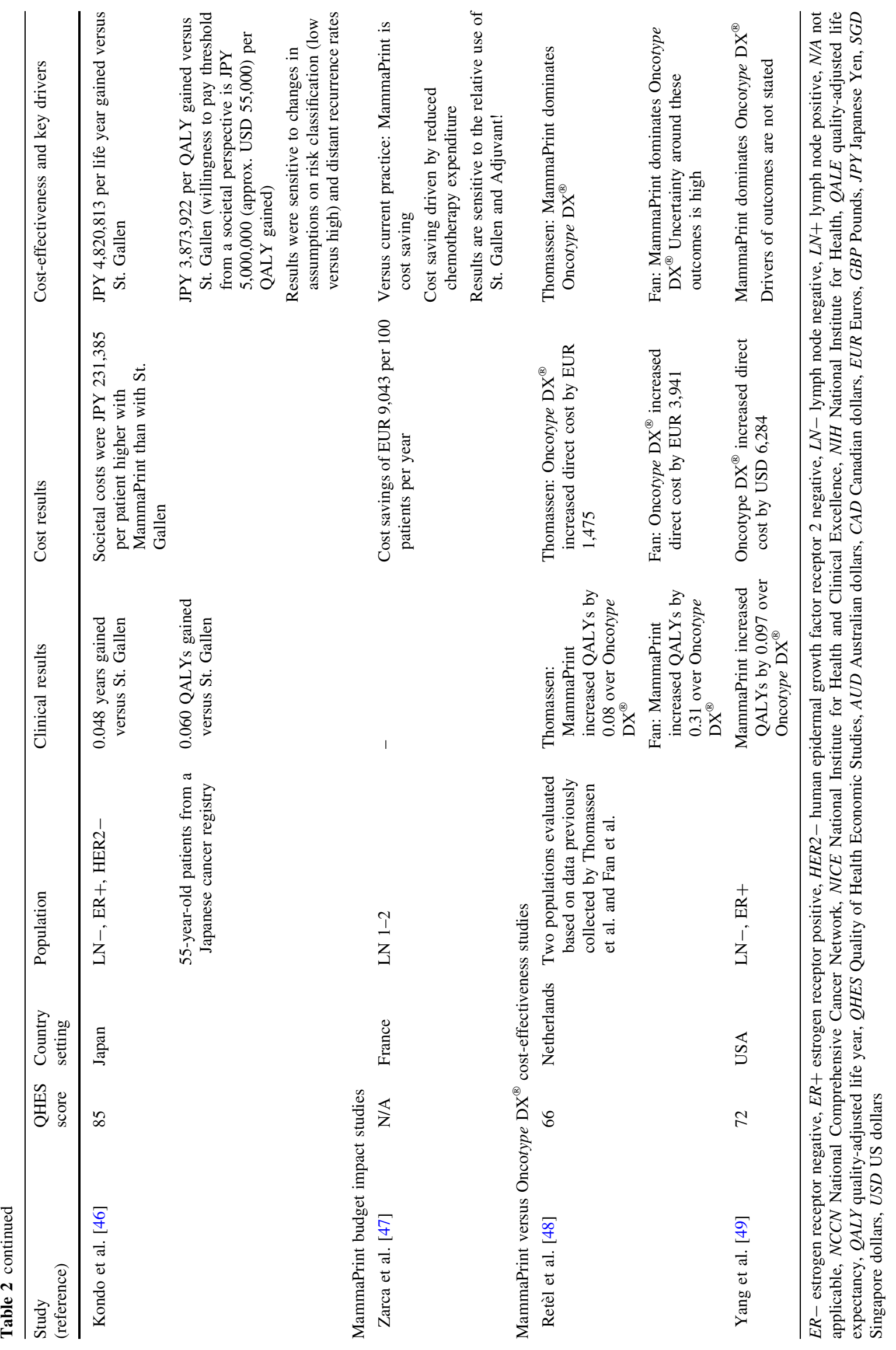


relative to usual care in four difference countries [26, 31, $34,37]$. In the published analyses, where costs have been accounted from a societal perspective, Oncotype $\mathrm{DX}^{\circledR}$ has been found to be cost saving [25, 29].

Most evaluations were conducted in lymph node negative ( $\mathrm{LN}-$ ) patients (with long-term outcomes based on the NSABP B-14 study [6]) but seven studies took into account lymph node positive $(\mathrm{LN}+)$ patients (taking data from the SWOG 8814 study [10]), either exclusively[30, 36, 37] or in a mixed cohort [27, 31, 33, 37, 38]. The analyses suggest that, while Oncotype $\mathrm{DX}^{\circledR}$ may be more cost-effective in $\mathrm{LN}$ - patients, it is also cost-effective in $\mathrm{LN}+$ patients and cohorts containing a mixture of $\mathrm{LN}-$ and $\mathrm{LN}+$ patients.

Cost-effectiveness evaluations of MammaPrint versus usual care

Four studies evaluated the cost-effectiveness of MammaPrint (Table 2) [43-46]. The 2010 article by Chen et al. [44] reported that MammaPrint was likely to be costeffective in the overall population, highly cost-effective in ER+ patients, but associated with reduced survival and quality-adjusted life expectancy (QALE) in patients with estrogen receptor negative (ER-) disease in the USA. These differences were driven by the proportion determined to be at high risk by the MammaPrint assay $(\mathrm{ER}+52 \%, \mathrm{ER}-94 \%)$. In the ER - group, MammaPrint spared only $6 \%$ of patients from receiving chemotherapy, compared with $12 \%$ in the ER+ analysis. The fall in chemotherapy usage in ER- patients led to reduced life expectancy due to increased rates of distant recurrence [50]. The authors noted that the results were very sensitive to variation in clinical input data to the model (particularly the proportion of patients with ER- disease), as well as the cost of chemotherapy and the cost of MammaPrint. Moreover, limited data on the predictive ability of the test meant that it was assumed that the benefits of chemotherapy in both low-risk and high-risk patients were the same.

Oestreicher et al. [43] reported negative outcomes (decreased survival, decreased QALE, and lower costs) for MammaPrint versus NIH clinical guidelines in a costeffectiveness evaluation in a mixed hypothetical population of early-stage breast cancer patients in the US setting. Poorer survival in the MammaPrint arm was driven by the sensitivity of the test, modeled as $84 \%$ in the base case analysis. The authors stated that a sensitivity of $95 \%$ is required, with specificity maintained at the current value, for MammaPrint to improve clinical outcomes. However, the analysis is subject to several notable limitations, including that the low risk/high assignation of patients using MammaPrint was assumed based on other studies (sensitivity and specificity estimates used to do this appear low) and that the analysis failed to distinguish between $\mathrm{ER}+$ and ER - patients.

In contrast, a more recent economic evaluation in the Netherlands (Retèl et al. [45]), showed improved survival and quality-adjusted survival for MammaPrint over both St. Gallen guidelines and Adjuvant!, and decreased costs over an approach based on St. Gallen guidelines. The analysis reported 20-year costs and outcomes for a hypothetical cohort of patients with $\mathrm{ER}+, \mathrm{LN}-$ disease, based on a Markov model populated with sensitivity and specificity data derived from a pooled analysis of 305 tumor samples from three previously reported validation studies of MammaPrint. Benefits were driven by fewer patients receiving unnecessary chemotherapy following MammaPrint testing compared with St. Gallen and Adjuvant! approaches to adjuvant therapy decision making. Similar findings were reported in the Japanese setting, where Kondo et al. [46] investigated the cost-effectiveness of MammaPrint versus St. Gallen criteria from a societal perspective using an adaptation of a model previously used to evaluate the cost-effectiveness of Oncotype $\mathrm{DX}^{\circledR}$. Projecting 10-year outcomes for a cohort of patients with $\mathrm{ER}+, \mathrm{LN}-$ disease, aged 55 years at baseline from a Japanese cancer registry, MammaPrint was associated with an improvement in QALE of 0.06 QALYs and an additional cost of JPY 231,385 per patient, leading to an ICER of $\sim$ JPY 3.9 million (USD 43,000) per QALY gained. Results were sensitive to changing assumptions around risk classification (low or high) and rates of distant recurrence.

\section{Cost-effectiveness studies comparing Oncotype $\mathrm{DX}^{\circledR}$ and MammaPrint}

Despite the lack of head-to-head clinical trial data or decision impact studies, two studies directly compared the cost-effectiveness of Oncotype $\mathrm{DX}^{\circledR}$ with MammaPrint, (Table 2) [48, 49]. Neither of these studies used the commercially available assays, but instead assayed expression of the same genes as in the commercial assays using their own methodologies. The authors acknowledge that there is significant uncertainty around the results and that the analyses should be repeated in the future, using a mixed treatment comparison approach or, ideally, data from a head-to-head trial of the two gene expression-profiling tests.

In the study by Yang et al. [49], MammaPrint was found to dominate Oncotype $\mathrm{DX}^{\circledR}$, both in terms of improving outcomes and reducing costs. There is, however, a lack of transparency regarding outcome drivers because only the final cost and QALE outcomes are reported. In the model, instead of modeling the two tests directly against each other, the two tests were individually compared with Adjuvant! and then outcomes compared. Furthermore, the 


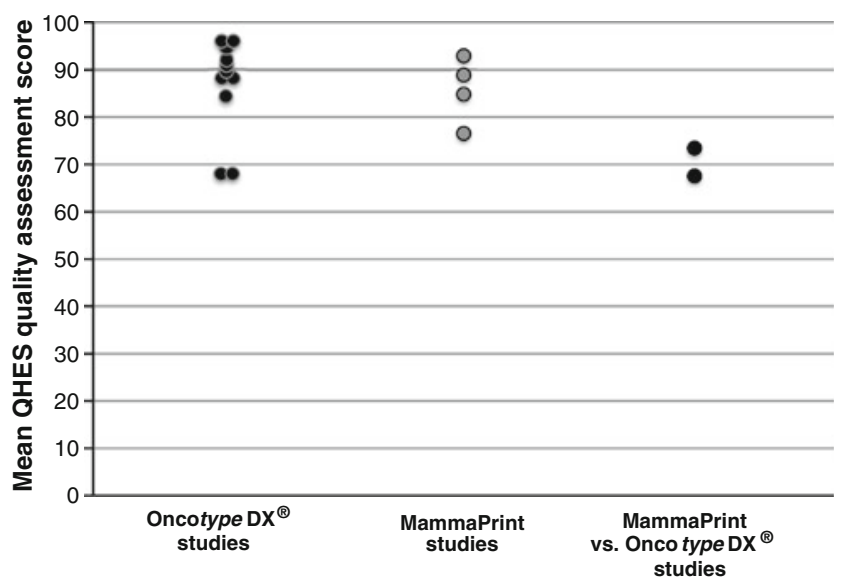

Fig. 2 Scatter plot of quality assessment of published, peer-reviewed cost-effectiveness evaluations of Oncotype DX ${ }^{\circledR}$, and MammaPrint. (The quality of studies supporting each gene expression test was assessed using the Quality of Health Economic Studies (QHES) instrument, generating a score out of 100, where higher scores reflect higher quality. Each circle represents the QHES score from a single study)

cohorts used in the two analyses were neither the same nor comparable. In the Oncotype $\mathrm{DX}^{\circledR}$ versus Adjuvant! analysis, $47 \%$ of patients were classified as high risk, while in the MammaPrint versus Adjuvant! analysis, $74 \%$ of patients were considered at high risk. Therefore, one would expect that more MammaPrint patients than Oncotype $\mathrm{DX}^{\circledR}$ patients would receive chemotherapy, and subsequently the MammaPrint patients would accrue higher costs than patients receiving Oncotype $\mathrm{DX}^{\circledR}$. The costs in the MammaPrint arm are, though, unexpectedly lower [51]. Moreover, Oncotype $\mathrm{DX}^{\circledR}$ patients with intermediate and high Recurrence Scores were spuriously grouped together to form a "high risk" group (as data suggests only around $50 \%$ of intermediate Recurrence Score patients receive chemotherapy). The noncomparable populations and the lack of transparency make the results and validity of this analysis difficult to interpret.

Retèl et al. [48] used retrospective data from two analyses of the sensitivity and specificity of a number of MGAs. In this study, the outcome measured influenced the method deemed most cost-effective. If the cost per QALY gained is the focus, then MammaPrint was most cost-effective. Concentrating, however, on the cost per life year gained, and Oncotype $\mathrm{DX}^{\circledR}$ has the highest probability of being cost-effective. Although providing more readily comparable datasets than that used in the Yang et al. study, a number of issues with this approach remain. Firstly, one retrospective study was small, containing only 26 tumor samples for assessment, taken from patients of whom few had received tamoxifen [52]. Secondly, the larger data set (295 samples) used data that was included in the development of MammaPrint; thus, increasing the likelihood that the MammaPrint predictions would be correct [53].
Budget impact studies

A total of four studies evaluated solely the budget impact of Oncotype $\mathrm{DX}^{\circledR}$ (as well as two that calculated both costeffectiveness and budget impact) and one evaluated the budget impact of MammaPrint. The analysis by de Lima Lopes et al. [29] used the model that was later used in the 2011 cost-effectiveness analysis by the same authors;[25]; however, clinical outcomes were not calculated in the first of these studies. This study found that, on a per patient basis in Singapore, Oncotype $\mathrm{DX}^{\circledR}$ was cost saving, mainly driven by reduced need for supportive care, and administration. Analyses in Canada and the USA similarly found Oncotype $\mathrm{DX}^{\circledR}$ to be cost saving [40, 42], while two analyses in the Irish setting reported approximate cost neutrality or cost saving [39, 41]. An UK-based study found that Oncotype $\mathrm{DX}^{\circledR}$ was cost saving as long as over $40 \%$ of the patients tested were found to be at low risk [30]. The major driver of cost savings in these budget impact analyses was the reduced number of chemotherapy prescriptions. A similar result was found in the budget impact study examining MammaPrint, where cost savings were reported in the French setting [47]. The magnitude of reduction in chemotherapy prescriptions in the budget impact analyses to date may be the key area of differentiation from the published cost-effectiveness studies (where chemotherapy sparing effects were more modest) in which MGAs appear to be generally cost-effective (but cost more than usual care). The only budget impact study in which the gene expression-profiling test was found to increase costs was a 2008 study of Oncotype DX $^{\circledR}$ in the Japanese setting [23].

\section{Evaluating study quality}

The quality of the identified analyses was high. The mean score for the studies comparing MGAs with current practice were both $86 / 100$ for the Oncotype DX $^{\circledR}$ and MammaPrint assays (Fig. 2). The most common area where studies did not meet QHES criterion was explicit discussion of bias (Appendix). A number of analyses also failed to fully describe the model constructed and the assumptions used. These shortcomings, however, were relatively minor and only five studies score $<80 / 100$.

The quality of the two studies comparing Oncotype $\mathrm{DX}^{\circledR}$ and MammaPrint was lower than those comparing with usual care, scoring 66 and 72. These two studies received lower ratings primarily as a result of the data sources used to determine the treatment effects. One study used randomized controlled trial evidence, but not from a head-to-head study of the two interventions, and the other used evidence from small retrospective studies, some of which was used to inform creation of the MammaPrint test. Moreover, chemotherapy allocation in high- and low-risk 
patient groups was based on assumption. These studies also received low scores as a result of limited sensitivity analyses and lack of transparency, as very limited information on the outcomes and drivers of outcomes were given.

\section{Discussion}

This review has collated the existing health economic analyses examining the use of MGAs in guiding adjuvant chemotherapy treatment in early-stage breast cancer. The cost-effectiveness of only two assays, Oncotype DX ${ }^{\circledR}$ and MammaPrint, has been assessed in published literature. The majority of the economic evaluations found that using gene expression profiling to guide adjuvant therapy was cost saving or cost-effective. This was the case for all studies comparing Oncotype $\mathrm{DX}^{\circledR}$ with current treatment covering a range of populations with ER+ early-stage breast cancer across a number of different countries. The cost-effectiveness profile of MammaPrint appears to be more complex. Two studies support cost-effectiveness in $\mathrm{ER}+$ populations, but in ER - patients and in a mixed $(\mathrm{ER}+, \mathrm{ER}-)$ population, the clinical outcomes were poorer following MammaPrint testing than with usual care. Two evaluations directly compared the cost-effectiveness of MammaPrint with Oncotype $\mathrm{DX}^{\circledR}$. Both analyses found MammaPrint to dominate Oncotype $\mathrm{DX}^{\circledR}$. Both, however, are beset with methodological shortcomings and appear to be at odds with the other published studies on the costeffectiveness of these gene-profiling tests. Budget impact analyses indicated that MGAs were likely to be cost neutral or cost saving in most settings (US, UK, Canada, Ireland, and Singapore for Oncotype DX ${ }^{\circledR}$, France for MammaPrint); the only exception was the analysis by Kondo et al. (2011), which reported that Oncotype $\mathrm{DX}^{\circledR}$ increased costs but was highly cost-effective in Japan.

Assay accuracy has a large effect on cost-effectiveness, since incorrect allocation to low risk groups increases the risk of distant recurrence as a result of under treatment. Furthermore, incorrect allocation to high risk reduces QoL and increases costs due to overtreatment with chemotherapy [43]. MGAs with significant clinical data to support their use were shown in health economic evaluations, to improve outcomes both by assigning patients to chemotherapy who would not have previously received treatment, and by sparing patients from the adverse effects of chemotherapy who are unlikely to benefit. Assays were most likely to be cost-effective in settings where a high proportion of earlystage breast cancer patients received chemotherapy and where chemotherapy was costly. In these settings, assays benefit patients by reducing the number of adverse events associated with chemotherapy and payers by reducing the cost of chemotherapy and associated care. Analyses showed that the cost-effectiveness of MGAs was sensitive to the risk profile of the population. If few patients are classified as being at low risk of distant recurrence, assay costs are not offset by reduced chemotherapy. Still, the literature supports the cost-effectiveness of MGAs in both $\mathrm{LN}-$ and LN+ patients. Published studies examined cost-effectiveness in Asia, Europe, and North America, indicating that clinical and cost benefits may be seen in a diverse range of healthcare settings worldwide.

Therapy allocation guided by either conventional approaches or Oncotype $\mathrm{DX}^{\circledR}$ was based on real-life data in five of the published analyses [26, 31, 32, 34, 37]. This highlights a limitation of studies on Oncotype $\mathrm{DX}^{\circledR}$ and MammaPrint that assign therapy directly from validation studies (a feature of evaluations comparing the two tests). In general, studies that assumed chemotherapy prescription rates produced more positive estimates of cost-effectiveness (often being cost saving) than those who relied on real-life data. The exception was the US evaluation in 2011, which showed Oncotype $\mathrm{DX}^{\circledR}$ testing to be dominant to decision making based on NCCN guidelines [32]. The NSABP B-20 study provided data in ER+, $\mathrm{LN}-$ patients and the SWOG 8814 trial provided data in $\mathrm{ER}+, \mathrm{LN}+$ patients both demonstrate the prediction of chemotherapy benefit provided by Oncotype $\operatorname{DX}^{\circledR}[9,10]$. The clinical validity of Oncotype $\mathrm{DX}^{\circledR}$ as both a prognostic indicator and a test predictive of likely chemotherapy benefit is supported by an evidence base of consistent results from multiple studies $[6,9,10,54,55]$. The evidence supporting other MGAs are not currently as strong [16].

The studies identified in this review were generally of high quality, as assessed by the QHES instrument. It should be acknowledged, however, that a general instrument such as QHES might have shortcomings for the assessment of cost-effectiveness analyses (Appendix). The development of a bespoke checklist focused on cost-effectiveness evaluation of diagnostic tests may be a valuable avenue of future research.

MGAs have the potential to help physicians make more informed treatment decisions by identifying patients at high risk of distant recurrence and patients who are likely to benefit from adjuvant chemotherapy. Similarly, identifying patients at low risk of distant recurrence who can be spared chemotherapy treatment has notable benefits, including avoidance of associated adverse events both in the short-term (captured in all models) and long-term (captured in one study). This review finds a consistent body of evidence supporting the cost-effectiveness of Oncotype $\mathrm{DX}^{\circledR}$ in informing chemotherapy treatment decisions regardless of local cost and local clinical practice.

In particular, for patients with ER+ early-stage breast cancer, the benefits negate the acquisition costs of Oncotype $\mathrm{DX}^{\circledR}$ and its use in these patients is encouraged. The 
literature suggests that MammaPrint is also likely to be cost-effective in this patient population. The body of evidence, however, is not as extensive and before encouraging its general use further research is needed to understand the influence of local treatment practices and to what extent this test predicts chemotherapy benefit. Future studies investigating head-to-head comparisons of MGAs would provide valuable insights into how these tests influence adjuvant therapy decision making, and would provide valuable data for future economic evaluations on the relative merits of tests in clinical practice in the years ahead.

Acknowledgments The authors would like to thank Juliette PlunFavreau of Genomic Health International, Geneva, Switzerland for her assistance with realization of this article. The authors are also grateful to Tallal Younis of Dalhousie University, Halifax, Nova Scotia, Canada for his constructive comments and review of the article. This study was supported by funding from Genomic Health International provided to Ossian Health Economics and Communications $\mathrm{GmbH}$. Only Barnaby Hunt and William Valentine, as employees of Ossian Health Economics and Communications $\mathrm{GmbH}$, received financial support for their contribution to this review article. No other authors received support for this study. This review was made possible by funding provided by Genomic Health International that facilitated project management and production of this manuscript through Ossian Health Economics and Communications GmbH.

Conflict of interest As employees of Ossian Health Economics and Communications $\mathrm{GmbH}$, Barnaby Hunt and William Valentine received remuneration from Genomic Health International for their participation in this study. Paolo Pronzato and Roman Rouzier have previously received remuneration from Genomic Health International. The following authors declare that they have no conflict of interest: Elisabeth Chéreau and Josh Carlson.

Open Access This article is distributed under the terms of the Creative Commons Attribution Noncommercial License which permits any noncommercial use, distribution, and reproduction in any medium, provided the original author(s) and the source are credited.

\section{Appendix}

\section{Congress databases}

The congress databases searched were: San Antonio Breast Cancer Symposium (SABC), American Society of Clinical Oncology (ASCO), European Breast Cancer Conference (EBCC), St. Gallen Oncology Conference, European Society for Medical Oncology (ESMO), European Cancer Organisation (ECCO), and International Society for Pharmacoeconomics and Outcomes Research (ISPOR).

\section{Evaluation of study quality}

The literature search identified 17 published manuscripts that were underwent quality assessment using the QHES instrument. Of these, 11 compared Oncotype $\mathrm{DX}^{\circledR}$ with current practice, four evaluated MammaPrint versus current practice, and two evaluated Oncotype $\mathrm{DX}^{\circledR}$ versus MammaPrint. Overall, the quality of the identified analyses was high. The mean score for the studies comparing MGAs with current practice were both $86 / 100$ for the Oncotype DX $^{\circledR}$ and MammaPrint assays (Fig. 2). The most common area where studies did not meet QHES criterion was explicit discussion of bias. A number of analyses also failed to fully describe the model constructed and the assumptions used. These shortcomings, however, were relatively minor and only five studies score $<80 / 100$.

The quality of the two studies comparing Oncotype $\mathrm{DX}^{\circledR}$ and MammaPrint was lower than those comparing with usual care, scoring 66 and 72. These two studies received lower ratings primarily as a result of the data sources used to determine the treatment effects. One study used randomized controlled trial evidence, but not from a head-to-head study of the two interventions, and the other used evidence from small retrospective studies, some of which was used to inform creation of the MammaPrint test. Moreover, chemotherapy allocation in high- and low-risk patient groups was based on assumption. These studies also received low scores as a result of limited sensitivity analyses and lack of transparency, as very limited information on the outcomes and drivers of outcomes was given.

\section{Limitations of the QHES instrument}

The studies identified in this review were generally of high quality, as assessed by the QHES instrument. It should be acknowledged, however, that a general instrument such as QHES might have shortcomings for the assessment of costeffectiveness analyses on diagnostic tests (or screening studies). For example, the evaluations included in this review generally followed a 2-step approach, with models designed to first evaluate therapy allocation, and second to simulate long-term outcomes. Both steps influence outcomes, and a general checklist might not be sensitive to differences between evaluations. The use of local decision impact study data versus assumptions from a validation study in step 1 is one example, particularly when the clinical data underpinning the second step of the analysis is sound. Moreover, the use of the "best available source" data (a criterion of the QHES) does not pick up on the relative merits of using, for example, randomized controlled trial data versus meta-analysis data versus assumption or investigator opinion, so long as, it represents the "best available" data. One additional point is that the QHES checklist does not address limitations in the modeling approach. Clearly, this may be difficult to achieve as part of a general checklist, but it would be a valuable tool for estimating the influence of the above points on the 
overall quality of cost-effectiveness modeling evaluation. The development of a bespoke checklist focused on costeffectiveness evaluation of diagnostic tests may be a valuable avenue of future research.

\section{References}

1. World Health Organization. Breast cancer prevention and control (2012) http://www.who.int/cancer/detection/breastcancer/en/. Accessed 2 July 2012

2. Coleman MP, Quaresma M, Berrino F et al (2008) Cancer survival in five continents: a worldwide population-based study (CONCORD). Lancet Oncol 9(8):730-756

3. Peasgood T, Ward SE, Brazier J (2010) Health-state utility values in breast cancer. Expert Rev Pharmacoecon Outcomes Res 10(5):553-566

4. Conner-Spady BL, Cumming C, Nabholtz JM, Jacobs P, Stewart D (2005) A longitudinal prospective study of health-related quality of life in breast cancer patients following high-dose chemotherapy with autologous blood stem cell transplantation. Bone Marrow Transpl 36(3):251-259

5. Barron JJ, Quimbo R, Nikam PT, Amonkar MM (2008) Assessing the economic burden of breast cancer in a US managed care population. Breast Cancer Res Treat 109(2):367-377

6. Paik S, Shak S, Tang G et al (2004) A multi-gene assay to predict recurrence of tamoxifen-treated, node-negative breast cancer. N Engl J Med 351:2817-2826

7. van 't Veer LJ, Dai H, van de Vijver MJ et al (2002) Gene expression profiling predicts clinical outcome of breast cancer. Nature 415(6871):530-536

8. Sotiriou C, Wirapati P, Loi S et al (2006) Gene expression profiling in breast cancer: understanding the molecular basis of histologic grade to improve prognosis. J Natl Cancer Inst 98(4):262-272

9. Paik S, Tang G, Shak S et al (2006) Gene expression and benefit of chemotherapy in women with node-negative, estrogen receptor-positive breast cancer. J Clin Oncol 24:3726-3734

10. Albain KS, Barlow WE, Ravdin PM et al (2009) Adjuvant chemotherapy and timing of tamoxifen in postmenopausal patients with endocrine-responsive, node-positive breast cancer: a phase 3 , openlabel, randomised controlled trial. Lancet 374(9707):2055-2063

11. Dowsett M, Goldhirsch A, Hayes DF, Senn HJ, Wood W, Viale G (2007) International web-based consultation on priorities for translational breast cancer research. Breast Cancer Res 9(6):R81

12. Goldhirsch A, Ingle JN, Gelber RD, Coates AS, Thürlimann B, Senn HJ, Panel members (2009) Thresholds for therapies: highlights of the St Gallen International Expert Consensus on the Primary Therapy of Early Breast Cancer 2009. Ann Oncol 20(8):1319-1329

13. Harris L, Fritsche H, Mennel R et al (2007) American Society of Clinical Oncology 2007 update of recommendations for the use of tumor markers in breast cancer. J Clin Oncol 25(33):52875312

14. NCCN Clinical Practice Guidelines in Oncology ${ }^{\mathrm{TM}}$ Breast Cancer, (Version 1.2011). http://www.nccn.org. Accessed 2 July 2012

15. National Institute of Health and Clinical Excellence. http://guid ance.nice.org.uk/DT/4. Accessed 2 July 2012

16. Paik $S$ (2011) Is gene array testing to be considered routine now? Breast 20(Suppl 3):S87-S91

17. Ravdin PM, Siminoff LA, Davis GJ, Mercer MB, Hewlett J, Gerson N, Parker HL (2001) Computer program to assist in making decisions about adjuvant therapy for women with early breast cancer. J Clin Oncol 19(4):980-991

18. Ofman JJ, Sullivan SD, Neumann PJ et al (2003) Examining the value and quality of health economic analyses: implications of utilizing the QHES. J Manag Care Pharm 9(1):53-61

19. Chiou CF, Hay JW, Wallace JF, Bloom BS, Neumann PJ, Sullivan SD et al (2003) Development and validation of a grading system for the quality of cost-effectiveness studies. Med Care 41(1):32-44

20. Marino P, Siani C, Bertucci F et al (2011) Economic issues involved in integrating genomic testing into clinical care: the case of genomic testing to guide decision-making about chemotherapy for breast cancer patients. Breast Cancer Res Treat 129:401-409

21. Hornberger J, Cosler LE, Lyman GH (2005) Economic analysis of targeting chemotherapy using a 21-gene RT-PCR assay in lymph-node-negative, estrogen-receptor-positive, early-stage breast cancer. Am J Manag Care 11(5):313-324

22. Lyman GH, Cosler LE, Kuderer NM, Hornberger J (2007) Impact of a 21-gene RT-PCR assay on treatment decisions in early-stage breast cancer: an economic analysis based on prognostic and predictive validation studies. Cancer 109(6):1011-1018

23. Kondo M, Hoshi SL, Ishiguro H, Yoshibayashi H, Toi M (2008) Economic evaluation of 21-gene reverse transcriptase-polymerase chain reaction assay in lymph-node-negative, estrogen-receptorpositive, early-stage breast cancer in Japan. Breast Cancer Res Treat 112:175-187

24. Cosler LE, Lyman GH (2009) Economic analysis of gene expression profile data to guide adjuvant treatment in women with early-stage breast cancer. Cancer Investig 27(10):953-959

25. de Lima Lopes G, Chien R, Hornberger J. Cost-benefit analysis of a 21-gene recurrence score for early-stage breast cancer in Singapore. Presented at ISPOR 4th Asia-Pacific Conference, Phuket, September 2010

26. Klang SH, Hammerman A, Liebermann N, Efrat N, Doberne J, Hornberger J (2010) Economic implications of 21-gene breast cancer risk assay from the perspective of an Israeli-managed health-care organization. Value Health 13(4):381-387

27. O'Leary B, Yoshizawa C, Foteff C, Chao C. Cost-effectiveness of the Oncotype DX assay in Australia: An exploratory analysis. Presented at ISPOR 4th Asia-Pacific Conference, Phuket, September 2010

28. Tsoi DT, Inoue M, Kelly CM, Verma S, Pritchard KI (2010) Cost-effectiveness analysis of recurrence score-guided treatment using a 21-gene assay in early breast cancer. Oncologist 15(5): $457-465$

29. de Lima Lopes G, Chien R, Hornberger JC (2011) Cost-benefit analysis of a 21-gene recurrence score for early-stage breast cancer in Singapore. Presented at 12th St. Gallen International Breast Cancer Conference, St Gallen, 16-19 March 2011

30. Hall PS, McCabe C, Stein RC, Cameron D (2012) Economic evaluation of genomic test-directed chemotherapy for early-stage lymph node-positive breast cancer. J Natl Cancer Inst 104(1): $56-66$

31. Holt SDH, Bennet H, Bertellii G, Valentine WJ, Phillips CJ (2011) Cost-effectiveness evaluation of the Oncotype $\mathrm{DX}^{\circledR}$ breast cancer assay in clinical practice in the UK. Poster presented at the 34th Annual San Antonio Breast Cancer Symposium, 6-10 December 2011

32. Hornberger J, Chien R, Krebs K, Hochheiser L (2011) US Insurance Program's Experience With a multigene assay for early-stage breast cancer. Am J Manag Care 17(5(Spec No)): e194-e202

33. Kondo M, Hoshi SL, Yamanaka T, Ishiguro H, Toi M (2011) Economic evaluation of the 21-gene signature (Oncotype DX) in lymph node-negative/positive, hormone receptor-positive early- 
stage breast cancer based on Japanese validation study (JBCRGTR03). Breast Cancer Res Treat 127(3):739-749

34. Lacey L, Chien R, Hornberger J (2011) Cost-utility of the 21-gene breast cancer assay (Oncotype $\mathrm{DX}^{\circledR}$ ) in the Irish healthcare setting. Poster presented at the 34th Annual San Antonio Breast Cancer Symposium, 6-10 December 2011

35. Paulden M, Franek J, Pham B, Krahn M (2011) Gene expression profiling for guiding adjuvant chemotherapy decisions in women with early breast cancer: a cost-effectiveness analysis of 1000 strategies for the provision of Adjuvant! Online, Oncotype DX and chemotherapy. Presented at ISPOR 16th Annual international Meeting, Baltimore, 21-25 May 2011

36. Vanderlaan BF, Broder MS, Chang EY, Oratz R, Bentley TG (2011) Cost-effectiveness of 21-gene assay in node-positive, early-stage breast cancer. Am J Manag Care 17(7):455-464

37. Lamond NW, Skedgel C, Rayson D, Lethbridge L, Younis T (2012) Cost-utility of the 21-gene recurrence score assay in nodenegative and node-positive breast cancer. Breast Cancer Res Treat 133(3): 1115-1123

38. Madaras B, Rózsa P, Gerencsér Z et al (2012) The impact of chemotherapeutic regimens on the cost-utility analysis of Oncotype DX Assay. Presented at EBCC 8, Vienna, 21-24 March 2012

39. Wilson E, McDonnell D, Gullo G et al (2012) Economic Impact of Oncotype DX assay in axillary node negative breast cancer, $(\mathrm{AXN}-\mathrm{BC})$ with positive hormone receptor $(\mathrm{REC}+)$ and normal HER-2 (HER2-). Presented at ESMO 2010, Milan, 8-12 October 2012

40. Hassan S, Mittmann N (2011) A cost benefit analysis of the 21-gene breast cancer assay within a Canadian healthcare system. Presented at 2011 ASCO Annual Meeting, Chicago, 3-7 June 2011

41. Lacey L, Hornberger J (2011) Economic evaluation of Onctoype DX to target chemotherapy use in lymph-node-negative, oestrogen-receptor-positive, early-stage breast cancer. ISPOR 13th Annual European conference, Prague, 6-9 November 2011

42. Ragaz J, Wilson KS, Wong H, Muraca G, Hryniuk W, Bajdik C (2011) Molecular classification with 21 Gene Assay (Oncotype DX $^{\circledR}$ ) Shows in 196,967 ER positive patients high frequency of low recurrence. Presented at Poster presented at the 34th Annual San Antonio Breast Cancer Symposium, 6-10 December 2011

43. Oestreicher N, Ramsey SD, Linden HM et al (2005) Gene expression profiling and breast cancer care: what are the potential benefits and policy implications? Genet Med 7(6):380-389

44. Chen E, Tong KB, Malin JL (2010) Cost-effectiveness of 70-gene MammaPrint signature in node-negative breast cancer. Am J Manag Care 16(12):e333-e342
45. Retèl VP, Joore MA, Knauer M, Linn SC, Hauptmann M, Harten WH (2010) Cost-effectiveness of the 70-gene signature versus St. Gallen guidelines and Adjuvant Online for early breast cancer. Eur J Cancer 46(8):1382-1391

46. Kondo M, Hoshi SL, Ishiguro H, Toi M (2012) Economic evaluation of the 70 -gene prognosis-signature (MammaPrint ${ }^{\circledR}$ ) in hormone receptor-positive, lymph node-negative, human epidermal growth factor receptor type 2-negative early stage breast cancer in Japan. Breast Cancer Res Treat 133(2):759-768

47. Zarca D, Stork-Sloots L, de Snoo F, Brink G, Dervaux Y, Boubli L (2009) French cost effectiveness study of the MammaPrint 70-gene signature in early-stage breast cancer patients. Presented at ECCO15, Berlin, Germany, 20-24 September 2009

48. Retèl VP, Joore MA, van Harten WH (2012) Head-to-head comparison of the 70-gene signature versus the 21-gene assay: cost-effectiveness and the effect of compliance. Breast Cancer Res Treat 131(2):627-636

49. Yang M, Rajan S, Issa AM (2012) Cost effectiveness of gene expression profiling for early stage breast cancer: a decisionanalytic model. Cancer 118(20):5163-5170

50. Buyse M, Loi S (2006) van't Veer L, et al; TRANSBIG Consortium. Validation and clinical utility of a 70-gene prognostic signature for women with node-negative breast cancer. J Natl Cancer Inst 98(17):1183-1192

51. Reed SD, Lyman GH (2012) Cost-effectiveness of gene expression profiling for early stage breast cancer: a decision-analytic model. Cancer 118(24):6298-6299

52. Thomassen M, Tan Q, Eiriksdottir F, Bak M, Cold S, Kruse TA (2007) Comparison of gene sets for expression profiling: prediction of metastasis from low-malignant breast cancer. Clin Cancer Res 13:5355-5360

53. Fan C, Oh DS, Wessels L, Weigelt B, Nuyten DS, Nobel AB, van't Veer LJ, Perou CM (2006) Concordance among gene-expressionbased predictors for breast cancer. N Engl J Med 355(6):560-569

54. Dowsett M, Cuzick J, Wale C et al (2010) Prediction of risk of distant recurrence using the 21-gene recurrence score in node-negative and node-positive postmenopausal patients with breast cancer treated with anastrozole or tamoxifen: a TransATAC Study. J Clin Oncol 28:1829-1834

55. Goldstein LJ, Gray R, Badve S et al (2008) Prognostic utility of the 21-gene assay in hormone receptor-positive operable breast cancer compared with classical clinicopathologic features. J Clin Oncol 26:4063-4071 